\title{
LA MUSICA EBRAICA TRA PERMESSI E DIVIETI NEI COMMENTARI MEDIEVALI
}

\author{
Enrico Fubini \\ Universidad de Torino
}

\begin{abstract}
RESUMEN
Nella storia del pensiero ebraico si trovano indicazioni che possono sembrare contraddictorie sul valore della musica e del canto. Divieti rigorosi si alternano a spiragli di permessi e a volte anche a esortazioni e prescrizioni vigorose circa l'uso del canto. Generalmente il pensiero di tendenza razionalistica tende a vietare la musica, soprattutto nei suoi usi profani, e non solo per il lutto perenne dovuto alla distruzione del tempio ma anche un caracttere intrinsecamente lascivo della musica stessa. Il pensiero mistico tende invece a considerare la musica e soprattutto il canto como una forma di elevazione spirituale insostituibile. Le Zohar, il grande testo del misticismo ebraico medievale, considera la musica como qualcosa di esoterico, di strettamente connesso con l'estasi mistica, con la profezia e comunque con la tensione verso el divino.
\end{abstract}

Palabras clave: Musica ebraica, Zohar.

\begin{abstract}
:
In the history of the Hebrew Thought there are some points about the value of music and chant wich can seem contradictory. Generally speaking, the rationalistic thought tends to forbid music, especially in profane uses because of its lascivious character. On the contrary, mystical thought tends to considerer music and chant as a way of spiritual rising. The Zohar, the great book of the Hebrew medieval mysticism, considers music as an esoteric thing related wich mysticism and prophecy.
\end{abstract}

Key words: Hebrew music, Zohar.

Quando Maimonide nel suo Mishné Torà elencava i famosi sei divieti riguardanti la musica, recepiva una lunga tradizione che risaliva almeno al Talmud e in cui veniva espressa una netta e inequivocabile ostilità nei confronti della musica e del canto. Molte sono le fonti, prima e dopo Maimonide e in pratica sino ai nostri giorni, per quanto riguarda le cerchie più ortodosse dell'ebraismo, che vietano categoricamente qualsiasi uso della musica. Dopo la distruzione del Tempio 
di Gerusalemme, un lutto perenne rende quanto meno non conveniente la musica, la quale, secondo una tradizione che risale alla Bibbia, è sempre espressione di gioia e di letizia. Se è indubbio che la distruzione del Tempio è strettamente connessa all'idea di un lutto che deve protrarsi sino al giorno della redenzione quando anche il Tempio verrà riedificato e che nell'attesa nessuna manifestazione di gioia può essere ammessa nel mondo ebraico, ci si è legittimamente chiesto se questo atteggiamento di ostilità nei confronti della musica sia solamente legato alla distruzione del Tempio o trovi invece anche delle motivazioni diverse e non legate ad avvenimenti contingenti come poteva essere la distruzione del santuario di Gerusalemme.' Va notato che Maimonide, elencando le proibizioni all'uso e all'ascolto di musica, nella sua rigida posizione, non fa cenno alla distruzione del Tempio, ma sembra riferirsi unicamente a un intrinseco carattere lascivo della musica stessa. L'ala più rigorista e razionalista del pensiero ebraico si è sempre riferita alle idee di Maimonide nel sostenere le proprie posizioni sulla musica le cui radici si ritrovano nei commentari quando interpretano l'enigmatica figura di Iuval, il mitico inventore degli strumenti di cui si parla molto ellitticamente nella Genesi. Iuval, come viene detto nella Genesi, fu il progenitore di tutti i suonatori di arpa e di cetra. Poche altre notizie vengono fornite su questo personaggio, figlio di Lémech e di Adà, una delle due mogli di Lémech; prima di luval viene ricordato il fratello laval che fu il progenitore di tutti coloro che abitano sotto le tende e conducono i greggi. L'ellitticità dei versetti in cui compare in modo così fuggevole il personaggio di Iuval, il primo musicista dell'umanità, ha sollecitato la fantasia dei commentatori i quali andando al di là della lettera del testo, hanno cercato di scavare nella sua personalità, per dirci qualcosa sulla natura e funzione della musica stessa.

La maggior parte dei commentatori interpretano negativamente la figura di Iuval, ravvisando nella sua attività di musicista le prove della sua natura idolatra; pochi commentatori vedono invece in lui il prototipo dell'uomo che cerca attraverso la musica un'elevazione spirituale e un contatto con Dio. Un midrash di Bereshit Rabba $(23,3)$ ci fornisce il prototipo di interpretazione negativa della figura di Iuval e dei suoi fratelli: Iuval avrebbe creato la musica per meglio servire gli idoli. «Dapprima [gli uomini ai tempi di Iaval] ingelosivano il Santo Egli sia benedetto di nascosto; dopo [ai tempi di Iuval] incominciarono a ingelosirlo pubblicamente». Anche la sorella Nahama non si sottrae a questo severo giudizio; infatti non mostrava migliori intenzioni in quanto «suonava il tamburello per il culto idolatrico» (lbid.) Anche secondo Abrabanel, Iuval non avrebbe avuto finalità molto migliori: attraverso la musica egli avrebbe aspirato a soddisfare la sua sete di onori e di dominio. Infatti il suo nome I $u v a l$ è da mettersi in relazione con hevel (vanità) allo stesso modo del nome di Abele il quale al pari di Iuval aveva scelto la professione di pastore per soddisfare il suo desiderio di dominio "portando le bestie al pascolo e picchiandole". Gli onori che egli ricercava non erano

1 Nel suo Mishné Torà Maimonide, rispondendo ad ebrei di Aleppo che gli chiedevano se era permesso ascoltare canti strofici degli Arabi accompagnati da strumenti a fiato, rispondeva, con riferimento anzituto agli strumenti, che si doveva ritenere tutti gli strumenti e tutta la musica fatta per procurare piacere come proibita. Inoltre aggiungeva in modo sistematico le altre principali proibizioni in ordine crescente di gravità:

1) Ascoltare canti su un testo profano, sia esso in Ebraico o in arabo.

2) Ascoltare un canto accompagnato da uno strumento.

3) Ascoltare un canto il cui contenuto linguistico sia sconveniente.

4) Ascoltare uno strumento a corde.

5) Ascoltare passaggi musicali su tali strumenti, bevendo vino.

6) Ascoltare una donna che canti o suoni. 
che vanità. Si comprende così perché luval è nominato accanto a suo fratello "progenitore di tutti i pastori".

Di grande interesse, sempre nell'ottica delle posizioni antimusicali, e per venire a tempi più vicini a noi, le osservazioni del Malbim, il quale, sulla scia delle altre posizioni negative, individua in Iuval il seduttore il quale si serve appunto della sua musica per esercitare le sue arti. Il Malbim si richiama al profeta Isaia $(23,15-16)$ per avvalorare la sua tesi sulle cattive arti di Iuval: «In quel giono Tiro cadrà in oblio per 70 anni, come i giomi di un re, e poi accadrà di Tiro come nella canzone cortigiana: "Prendi la cetra (kinnor), fai il giro della città, cortigiana dimenticata; suona bene, moltiplica i tuoi canti affinché ci si ricordi di te!". Così commenta il Malbim i versetti di Isaia: «In effetti chi vive nell'errore usava intonare i canti della cortigiana, sia nei festini che nei templi dell' $i$ dolatria e anche se la musica è stata ricondotta a fini più nobili, il primo compositore se ne è servito per sedurre il cuore delle donne. Questo è il motivo per cui lo strumento si chiama ugav come nell'espressione shir "agavim (canto d'amore)». (Erez Hemdah, 42) Non mancano commenti più indulgenti nei confronti di Iuval, come il Keli Yakar di Schlomo Efraym e spesso vengono capovolti i precedenti giudizi dal momento che sono portati a vedere nella sua arte uno strumento di elevazione spirituale per lodare il creatore e per riportare tra gli uomini la Presenza divina, o per venire a tempi più vicini a noi «per riportare l'armonia divina che è interiormente spezzata» (Hirsch).

I testi della mistica ebraica in genere considerano in modo del tutto positivo la figura di Iuval e quindi la musica stessa, concepita per lo più come un mezzo privilegiato di elevazione a Dio. Nei testi hassidici abbondano le osservazioni sulla musica come arte necessaria all'uomo per il suo innalzamento spirituale; il legame profondo tra pastorizia e musica è sempre al centro delle speculazioni dei pensatori hassidici i quali, andando essi pure al di là della lettera del testo biblico, interpretano in chiave mistico-naturalistica l'accenno di Bereschit ai due fratelli Iaval e luval ${ }^{2}$. Non è qui il caso di soffermarci sui pensatori hassidici che ci porterebbero fuori dall'ambito storico che ci eravamo prescelti. D'altra parte già nel Talmud si parla spesso di musica o come canto della Torà o in senso astratto come canto degli angeli e degli astri e ciò spiega il motivo per cui si trovano passi in cui la musica viene vietata in modo categorico altemati ad altri passi in cui musica come canto della Torà viene prescritta come un obbligo religioso imprescindibile. Certo non si tratta mai del canto come viene inteso normalmente nella tradizione occidentale, cioè come libera espansione del cuore in una melodia che sgorghi naturalmente, quando i sentimenti urgono. Quando nel Talmud si parla di canto lo si intende in tutt'altra accezione. L'uso del canto deve intendersi anzitutto solo in una comice di sacralità. Nella mentalità ebraica sacro può essere qualsiasi circostanza della vita che si svolga nell'ambito della quotidianità, ebraicamente intesa. Sacro perciò possono essere i numerosi momenti che scan-

2 Jossef Yitzak Schneerson così scrive nel suo Sefer Haniggunim (III, p. 13): «La vicinanza dei due versetti "progenitore di coloro che abitano sotto le tende" e "progenitore di coloro che suonano l'arpa e la lira" ci insegna che così come la necessità del pascolo è un bene per l'umanità anche la musica e gli strumenti musicali sono necessari al nostro mondo. Si giustifica così, verosimilmente, la tradizione musicale de i Hassidim, ispirati alle melodie dei pastori che esprimono per mezzo di queste i loro stati d animon. Ancor piu significative dal punto di vista dell ideologia hassidica le affermazioni di Dov Baer di Lubavitch, il quale, nel suo Torat hayn scriveva: «Bisogna anzituto comprendere cio che si deve intendere per "progenitore di tutti coloro che suonano l'appa il flauto"; si vuole indicare l'orgine di tuti i musicisti che hanno raggiunto un alto grado di spiritualità.... In effetti la musica è al tempo stesso un'elevazione e un annichilimento di ogni essere; infatti qualsiasi elevazione dal basso (materialità) verso l'allo (spiritualità) devo passare attraverso un annichilimento dell' essere che non si può realizzare che attraverso la musicas. 
discono la giornata, la settimana, i mesi della vita ebraica, la nascita, la circoncisione, il matrimonio e soprattutto il sabato, che in una famiglia ebraica religiosa risuona di canti che coniugano la vita famigliare con quella comunitaria in una ricorrenza che scandisce la sacralità del tempo entro cui si svolge la vita dell'ebreo. Il canto perciò, nell'ambito del sacro ebraicamente inteso, è non solo ammesso ma per certi aspetti anche prescritto. Il canto non è mai considerato come fine a se stesso, ma sempre in relazione ad un testo di cui rappresenta in qualche modo il completamento, la piena attuazione. Perciò il canto della Torà, eseguito secondo le prescrizioni rituali, cioè nelle modalità precise della cantillazione secondo la tradizione antichissima che pare risalga ai profeti Esra e Nehemia è il modello di canto per eccellenza. Famoso è il passo del Talmud che si riferisce per l'appunto alla lettura del Pentateuco in cui si afferma perentoriamente che «colui che legge la Torà senza cantare 0 che studia senza le modulazioni prescritte il Testo» è come se Dio «gli avesse dato precetti non buoni e leggi che non gli permettono di vivere» ${ }^{3}$. Non è il caso di entrare nel merito di ciò che può significare dal punto di vista tecnico cantare o intonare un testo come potevano essere non solo la Torà ma anche la Mishnà o il Talmud stesso. Ciò che è importante qui notare è l'obbligatorietà inerente a tale canto, ma purché si esplichi entro le norme che lo regolano: perciò non come effusione di spontanea religiosità ma come interpretazione corretta del testo, come piena esplicazione del suo significato, in tutte le sue componenti. In un altro passo talmudico si afferma: «Colui che recita, fuori di proposito un versetto della Bibbia in un banchetto, nuoce al mondo, perché allora la Torà si copre di un sacco e si presenta dinnanzi al Santo Benedetto Egli sia e dice: "Padrone del mondo, i Tuoi figli hanno fatto di me un liuto su cui suona gente frivola..., ${ }^{4}$. Di qui si ricava l'importanza della corretta intonazione del testo secondo una rigida normativa e non secondo una libera espressione del cuore.

La preferenza dimostrata per la musica vocale nella tradizione ebraica, almeno per quanto riguarda l'epoca sinagogale, dopo la distruzione del Tempio, si spiega non soltanto per il motivo a cui si è già accennato del lutto per la distruzione, ma soprattutto per la costante presenza di un testo che sorregge il canto e lo giustifica non come ornamento fine a se stesso. Infatti la linea melodica con cui viene intonato il testo non rappresenta, come avviene per lo più nella tradizione cristiana, un'aggiunta facoltativa per rendere più piacevole e più fruibile il testo, ma come un elemento indispensabile alla sua comprensione, parte integrante di esso. Per questo motivo il Talmud afferma che non cantare la Torà equivale a violare le leggi di Dio: la mancanza del canto equivale ad una mutilazione del testo o comunque ad una sua distorsione ${ }^{5}$.

3 Talmud Babli, Meghillà 32 a)

4. Ibid. Sanhedrin 101 a.

5 Per entrare nel vivo del meccanismo entro cui si dispiega la cantillazione biblica, la musica ebraica per eccellenza, può essere utile ripercortere il cammino che ha portato i Masoreti (coloro che trasmettono la tradizione) a fissare attorno al decimo secolo il testo biblico con i segni vocalici e con quelli musicali (te' amim) quali ancora oggi si ritrovano identici nel testo biblico. I te'amim, insieme alle vocali facevano parte di quella tradizione orale che veniva tramandata di generazione in generazione. La fissazione di questi elementi fa parte di quel processo di definizione di una tradizione che avrebbe per principio dovuto rimanere orale ma che la situazione diasporica ba imposto di fissare pena il rischio di andare perduta. Tutto ciò può sembrare in contrasto con il fatto che oggi la Bibbia viene intonata in modo diverso da comunità a comunità. Ma ciò è permesso e previsto proprio dal tipo di segno musicale fissato dai Masoreti, i te'amim, che definiscono in modo molto preciso la scansione ritmica del discorso, o legami tra le parole e le parti del discorso, le sue pause e i suoi respiri, mentre del tutto indeterminato rimane l'arco melodico entro cui ogni frase o versetto viene inglobato. Infatti nulla in proposito ci dicono i te amim, piccoli segni grafici che per il loro valore non possono essere avvicinati ai neumi medievali della canto gregoriano, i quali invece ci danno in modo embrionale una traccia della melodia. 
Il confine tra ciò che è vietato e ciò che è permesso è dunque assai stretto, anche se dal punto di vista concettuale i limiti sono fissati in modo preciso. La cantillazione biblica pertanto risulta non soltanto dal punto di vista storico il nucleo centrale e di maggior rilievo del canto ebraico attraverso i secoli ma anche dal punto di vista concettuale i testi ebraici, soprattutto quelli medievali, quando parlano di canto, di quello permesso, alludono sempre alla cantillazione e su di essa e sui segni che la definiscono e la rappresentano, i te'amim, possiamo trovare le più interessanti riflessioni dei mistici. Non di rado questi piccoli segni, insieme a quelli vocalici vengono interpretati come la parte più segreta e occulta e quindi come la più importante del testo, in qualche modo la sua anima, mentre le lettere rappresentano il corpo.

Nello Zohar, testo di provenienża medievale del XIII secolo, attribuito a Mosé de Leon, i riferimenti alla musica e al canto sono numerosissimi: questo difficile e spesso ermetico testo ha alle sue spalle una lunga tradizione mistica che affonda le sue radici anche nello stesso Talmud, almeno in quelle parti, spesso trascurate, in cui già affiora un pensiero mistico. Numerosissimi sono i passi dello Zohar in cui si parla di musica e di canti. Ma per capire il senso di queste allusioni bisogna por mente al fatto che generalmente si parla di musica in senso metaforico: si tratta per lo più di canti degli angeli, di canti celestiali, canti delle stelle 0 anche canti degli uomini, ma sempre canti in onore di Dio e molto raramente si tratta di musica nel senso odierno del termine. Uno dei problemi che compare nello Zohar e in molti altri testi mistici medievali ${ }^{6}$ è il valore da attribuirsi ai te'amim, cioè ai segni che indicano gli accenti musicali che compaiono nel testo masoretico della Bibbia. Dato per scontato che tutto ciò che compone il testo è rivelazione, qual'è l'ordine di priorità rispetto alla densità significativa per quanto riguarda tutti i tipi di segni di cui si compone il testo? Lo Zohar, commentando il versetto del libro di Daniele «l maestri di saggezza risplenderanno come lo splendore del firmamento» (Dan., 12,3) così afferma:

Lo splendore equivale ai segni della melodia. Le consonanti e le vocali li seguiranno nei loro movimenti, si muoveranno al loro seguito come soldati dietro al loro re. Il corpo sono le consonanti, il soffio vitale sono le vocali e insieme esse si spostano, accompagnando i segni della cantillazione e fermandosi quando questi ultimi si spostano. Quando la melodia dei segni si sposta, consonanti e vocali si spostano; quando si ferma, si fermano anch'esse, non si muovono più e si sottomettono alla suâ fermata. «I maestri di saggezza risplenderanno» allude alle consonanti e alle vocali; «come lo splendore dei cieli» allude al dispiegamento della melodia dei segni; «lo spazion allude al dispiegamento della melodia e dei suoi segni... (Zohar, Bereschit, 15b).

Questo passo, nel consueto linguaggio spesso ermetico e allusivo dello Zohar, mostra tuttavia con sufficiente chiarezza che i segni della cantillazione, i te'amim rappresentano l'elemento più importante nel corpo della scrittura, quello da cui dipende tutto il resto. La melodia dunque come elemento primario, quello che dà senso e spessore significativo al testo stesso. Da questo passo si ricava l'idea che la musica o meglio l'elemento musicale della Torà sia in qualche modo privile-

6 Si veda ad esempio nel testo di Jeuda Ha-Lewi, poeta e filosofo dell'undicesimo-dodicesimo secolo, Il re dei Khazari, la discussione tra maestro ed allievo sul valore dei te'amim e il maestro conclude indicando proprio nei te'amim la tradizione orale $\mathrm{e}$ il valore d'interpretazione del testo propria alla tradizione orale. 
giato rispetto alla lettera, 0 , in altre parole, che esso ci aiuta a scoprire ciò che sta al di là della lettera 0 è nascosto nella stessa lettera.

Uno dei motivi ricorrenti nello Zohar è che attraverso il canto si rende onore a Dio e che così fanno gli angeli, le schiere celesti e così fanno gli uomini. perché «Dio si delizia dei canti e degli inni». L'elemento estetico non è escluso da tali canti di lode; infatti «chiunque desideri lodare Dio con i canti dovrebbe avere una voce gradevole in modo che chi ascolta possa trarne piacere; altrimenti non deve procedere con il canto. Si raccomandava inoltre che i leviti si ritirassero dal servizio all'età di cinquant'anni, proprio perché a quell'età la voce incomincia a venir meno e a non essere più gradevole all'ascolto» (Zohar, Bereshit, 249 b). Il canto pertanto porta armonia e concilia gli opposti: le schiere celesti, gli angeli cioè e gli uomini cantano all'unisono e con armonia le loro lodi a Dio:

Quando coloro che cantano qui di sotto, appartenenti alla tribù di Levi e tutti coloro che furono santificati e che entrarono al loro servizio, e i due cori, quello del cielo e quello della terra, entrambi sono stati santificati e cantarono in armonia cosicché i mondi erano all'unisono ed un unico Re abitava sopra ad essi.... (Zohar, Shemot, sez. 2, 19 a).

Il canto armonizza anche la destra con la sinistra, cioè la giustizia con la misericordia, i due attributi più importanti della divinità. Ma il canto di cui si parla nello Zohar è tanto lontano da qualsiasi accezione edonistica che viene per lo più associata allo studio e alle veglie notturne del Re David. La notte è sempre considerata come il tempo elettivo da dedicare al canto e allo studio, cioè ad un'attività contemplativa, mentre il giorno è dedicato al lavoro e ad ogni tipo di operosità. La notte è dunque un momento privilegiato nel rapporto dell'uomo con Dio ed è per questo motivo che la notte è fatta per elevare canti di lode:

... le lodi che s'innalzavano verso di Lui nella notte rappresentano la lode perfetta. Vieni e osserva: nell'ora in cui i figli d'Israele erano rinchiusi nelle loro case, mentre il Santo Benedetto Egli sia faceva morire i primogeniti egizi, essi cantavano l'Hallel e le lodi in Suo onore. Anche il Re David si alzava nel bel mezzo della notte, e se tu pensi che egli restasse seduto o sdraiato mentre pronunciava i suoi canti di lode, ti inganni! Egli era sicuramente in piedi per darsi al canto e alle lodi della Torà (Zohar, Lech Lecha 82 b).

Ricorre molto spesso nello Zohar l'immagine della notte associata al Re David e ai canti. Si legge ancora:

... Davide conosceva questo momento propizio; la tradizione ci ha insegnato infatti che sopra il suo letto era appesa una cetra, e nel mezzo della notte, il vento del Nord vi soffiava sopra. Allora David si alzava e si dava ai canti e alle lodi. E proprio ciò che testimonia il passaggio: Svegliati, anima mia, svegliatevi o liuto e cetra! Io sveglierò l'alba (Salmi, 57, 9)... Tutte le notti il Santo Benedetto Egli sia e tutti i saggi dell'Eden, ascoltavano David nel cuore della notte... (Zohar, Midrash HaNeelam, 13 b, c).

Se la preghiera, il canto di lode, lo studio in altre parole, rappresenta il mezzo più alto che l'uomo possiede, quando ne è capace, per rapportarsi a Dio, questo si deve al potere primordiale del suono, simboleggiato nello Zohar dal suono dello Shofar, il corno d'ariete, carico di una comples- 
sa simbologia in tutta la letteratura biblica. Nello Zohar, il suono dello Shofar viene avvicinato alla stessa creazione nel suo atto primordiale: «Così Elohim creò il cielo, vale a dire che creò il suono dello Shofar» (Zohar, Bereshit, 29 b). Questo passo vuole alludere al concetto metaforico secondo cui il canto ha un potere primordiale al punto che nella sua espressione divina viene a configurarsi come l'atto creatore per eccellenza; il canto degli uomini si avvicina in qualche modo al canto divino, al suono emesso da Dio nel momento della creazione.

L'immagine del vento del Nord che spira di notte e fa risuonare l'arpa di David ritorna anche in altri autori della letteratura mistica e kabbalistica medievale, e a volte si arricchisce di altri significati ancora. Il kinnor, l'arpa è piena di cavità e di buchi e perciò risuona quando soffia il vento; così è anche il corpo umano che risuona, cioè profetizza quando scende su di lui la Shekinah (lo spirito divino). Vi è dunque un'analogia tra la profezia e la musica il che viene messo più volte in rilievo dai mistici. L'uomo è così paragonato ad uno strumento musicale e la profezia al suono che esso emette quando è investito dal soffio divino. Questo legame tra musica e profezia è indicato in vari modi da Abraham Abulafia, famoso mistico ebreo del XIII secolo e dai mistici della sua scuola come R. Isaiah ben Joseph, Jitzchak ben Ja'aqov ha-Kohen e altri ancora. R. Isaiah scrive nel suo libro Otzar ha-Chokhmah (Il tesoro della sapienza):

Sappi che il profeta, quando desidera profetizzare, deve prima

isolarsi per un determinato periodo ed eseguire le sue abluzioni. Dopo si mette in un luogo speciale e poi fa venire musicisti di vari strumenti che suonano per lui e cantano cantici spirituali, ed egli affronterà alcuni capitoli di questo libro.... Poi i musicisti cominceranno a suonare... ${ }^{7}$

Qui la musica appare nella funzione per così dire di coadiuvante della profezia, ma in altri autori la musica è considerata come un elemento strettamente connesso alla presenza della Shekinah:

Qual'è il servizio nel nome del Signore? Si deve dire che è quello del canto. Questo cantare era una cosa grande e tremenda "una corona di gloria, uno splendido diadema" (Isaia 28, 5). Per mezzo del canto melodico, sia vocale che strumentale, l'anima è risvegliata e il Santo Spirito splende in essa ed essa viene elevata, e comprende cose molto più elevate di quanto non abbia mai compreso. Questo canto degno di lode è il suono che proviene dalla bocca dei musici con timore, riverenza e santità, salendo e scendendo, estendendosi e abbreviandosi come sè provenisse dal canto degli angeli del cielo. ${ }^{8}$

Pur nelle differenze tra autore e autore ciò che è comune a tutti i testi dei mistici, compreso lo Zohar, è la considerazione della musica come qualcosa di esoterico, di strettamente connesso con l'estasi mistica, con la profezia e comunque con la tensione verso Dio. Anche l'entrata del Sommo Sacerdote nel Santo dei Santi è vista come simbolo di un'esperienza connessa con la musica da Joseph ben Shlomo Ashkenazi, mistico del XIV secolo:

7 Citato in Moshé Idel, The Mystical Experience in Abraham Abulafia, State University of New York Press, 1992. (Trad. italiana: L'esperienza mistica in Abraham Abulafia, Jaca Book, 1992, p 84).

8 Ibid., p. 86. 
Le lettere sgorgano nei sentieri attraverso la via della musica e questo è il segreto degli accenti della melodia della Torà (te'amim) perché essi entrano ed escono con i suoni del canto. Il segreto di questo è il campanello d'oro e la melagrana con i quali il Sommo Sacerdote era solito entrare nel Santo dei Santi, così che il suono potesse essere udito. Di questo tu comprenderai il segreto del Santo Spirito che risiede nei profeti sotto forma di musica ${ }^{9}$

Nelle correnti mistiche dell'ebraismo il problema dei divieti connessi alla pratica musicale e all'uso scorretto della musica stessa è del tutto superato. Si affaccia una prospettiva del tutto nuova che porterà più tardi anche ad una nuova prassi musicale, legata al diffondersi del movimento hassidico nel XVIII secolo. Pertanto è bene tener presente che nell' ebraismo le correnti mistiche non sono mai del tutto separate da quelle razionalistiche. Molti spunti del pensiero mistico, anche riguardante la musica, si trovano già nel Talmud e infatti non è infrequente che il pensiero mistico si appoggi a versetti sia biblici che talmudici a sostegno delle proprie tesi. Caratteristica del pensiero mistico ebraico, come ha giustamente messo in rilievo $\mathrm{G}$. Scholem è di non essere mai scivolato nell'eresia e di non aver mai messo in forse il valore e i principi dell'ortodossia. Anche per quanto riguarda la musica probabilmente i divieti al riguardo evidenziati dall'ebraismo ortodosso, non sono stati messi in forse ma dati per sottintesi. Tutto ciò che afferma l'ebraismo mistico sulla musica non è in contraddizione con l'ortodossia ma si affianca e si aggiunge ad essa, non contraddicendo ma integrando la prospettiva razionalistica.

Enrico Fubini

Strada S. Vito, 64

10133 Torino (Italia)

9 Ibid. p. 93. 